\title{
The Archives of Internal Medicine
}

\begin{tabular}{lrr}
\hline \hline Vol. XXI & FEBRUARY. 1918 & No. 2 \\
\hline
\end{tabular}

\author{
HEART-BLOCK \\ II. TRANSIENT COMPLETE hEART-BLOCK With NUMEROUS \\ STOKES-ADAMS ATTACKS * \\ FRANK N. WILSON, M.D. \\ AND \\ G. CANBY ROBINSON, M.D. \\ ST. LOUIS
}

Although syncopal attacks are fairly common in cases of complete heart-block, they do not, as a rule, occur with sufficient frequency in any given case to allow the cardiac mechanism during an attack to be graphically recorded. Polygrams have been obtained during such attacks by Lewis, ${ }^{1}$ and Williams ${ }^{2}$ has obtained an electrocardiogram during an attack in one instance. The following case is of interest because electrocardiographic records were obtained during several Stokes-Adams attacks, and also as an example of transient complete heart-block.

\section{REPORT OF CASE}

History.-Mrs. N: M., an American housewife, aged 48, entered the Barnes Hospital, May 10, 1917, on the advice of her family physician, Dr. George Park. She complained of fainting spells, vomiting and pain in the epigastrium and back. The family history was unimportant. She had had measles, mumps and a number of attacks of severe sore throat during childhood; but had been otherwise well until about ten years before, when she began to suffer from palpitation of the heart and slight dyspnea on exertion, both of which symptoms had continued up to the onset of the illness for which she entered the hospital. She had never had a severe attack of cardiac decompensation. She had had several attacks of severe pain in the epigastrium and back with fever and vomiting, and one such attack was accompanied by jaundice.

Present Illness.-About April 18, 1917, she became ill with pain in the epigastrium and back similar to that which she had experienced in previous attacks. At this time she also had nausea and vomiting and some fever. Her general condition remained about the same until the night of May 9. At about midnight of this date she became nauseated and asked for water. Her husband went to procure this, and when he returned he found her unconscious. Her pulse was weak and irregular. A dash of cold water in the face revived her. During the

* Submitted for publication Sept. 1, 1917.

* From the Department of Internal Medicine, Washington University Medical School. 1915

1. Lewis, T.: Lectures on the Heart, New York, Paul B. Hoeber Company,

2. Williams: Personal communication. 
course of the night she had ten or twelve such attacks, and several more occurred during the next forenoon. About 1 p. m., May 10, 1917, she was brought to the hospital. She was then having a syncopal attack every one or two minutes. These attacks were described by the intern in charge about as follows: Each attack was preceded by a cessation of the heart sounds and of the pulse, which did not return for from three to ten seconds. Just before the pulse returned, after the longer pauses, the patient became cyanotic and very dyspneic, the eyes turned upward and she became unconscious. At the end of the longer attacks there was some twitching of the extremities and occasionally of the whole body. The heart sounds and the pulse then returnd, the patient quickly regained consciousness and the cyanosis and dyspnea disappeared.

Physical Examination.-Aside from these attacks the examination of the patient revealed little that was abnormal. The cardiac dulness extended $3 \mathrm{~cm}$. to the right and $10 \mathrm{~cm}$. to the left of the midline. On auscultation there was a systolic murmur, heard best along the left border of the sternum, but heard distinctly at the apex and transmitted into the axilla. There was no edema of the ankles, ascites or hydrothorax. The liver extended $3 \mathrm{~cm}$. below the costal margin and there was slight general abdominal tenderness. The temperature reached $100.5 \mathrm{~F}$. on the day of admission, but was normal thereafter. The remainder of the physical examination and the laboratory examinations, including the Wassermann reaction, were negative.

\section{GENERAL SURVEY OF THE GRAPHIC RECORDS}

A general survey of the graphic records and the course of the patient's illness will be given first, after which the records will be described in detail. The electrocardiograms taken May 10 shortly after the patient entered the hospital and while she was having syncopal attacks, revealed the presence of complete auriculo-ventricular dissaciation with frequent periods of ventricular standstill lasting from seven to eleven seconds, and corresponding to the syncopal attacks. About one-half hour later these periods of ventricular standstill began to be interrupted in the electrocardiograms by abnormal ventricular complexes, and at about the same time the syncopal attacks ceased. During all of this time the ventricular rate between periods of ventricular standstill was approximately 90 per minute, while the auricles beat uninterruptedly at a rate of about 120 per minute. About one hour after the first curves were taken, however, single normal ventricular complexes began to interrupt the periods of ventricular standstill and these periods became comparatively short. One-half hour later the short periods of high ventricular rate with complete dissociation had disappeared and a partial heart-block of high grade was present.

On the following day, May 11, complete dissociation returned for a time, but there were no periods of ventricular standstill and the complete dissociation quickly gave place to a $2: 1$ block. The partial block then became gradually less marked until May 14, when the only sign of defective conduction which remained was a prolongation of the $\mathrm{P}-\mathrm{R}$ interval which measured 0.31 second. Thereafter, the improve- 
ment in conduction was less rapid, but by May 25 the P-R interval had decreased to 0.20 second. During this time there was also a rapid improvement in the patient's general clinical condition.

The general picture, then, was one of complete dissociation with a rapid idioventricular rate and ventricular standstill causing StokesAdams syndrome. This was followed by improvement associated with a rapid decrease in the grade of the heart-block.

\section{DETAILED DESCRIPTION OF THE GRAPHIC RECORDS}

Figure 1 is an example of the electrocardiograms that were obtained at about 2 p. m. May 10 , when the patient was having frequent syncopal attacks. It begins with a period of complete $\mathrm{A}-\mathrm{V}$ dissociation during which the ventricular rate is 89.3 and the auricular rate 120 per minute. This period of complete dissociation is followed by a period of ventricular standstill lasting 9.06 seconds. During this period there is a gradual acceleration of the auricular rate, probably due to anemia of the vagus center, or possibly to the fall of blood pressure. The period of ventricular standstill is terminated by a period of complete dissociation exactly like that at the beginning of the figure. From the examination of a number of tracings similar to this one it became apparent that there was a fairly constant time interval between the first ventricular complex following a period of ventricular standstill and an auricular beat preceding it. This relationship was sufficiently constant to warrant the conclusion that the periods of ventricular standstill were terminated by an effective impulse passing from the auricles to the ventricles.

Figure 2 is an example of the electrocardiograms obtained about 3:00 p. m., May 10, at about the time that the syncopal attacks ceased. It is very similar to Figure 1, except that in it the long period of ventricular standstill was interrupted by the occurrence of ventricular contractions yielding complexes suggesting stimulus production within the left ventricle. The relationship of the first normal ventricular complex following the long pause to the preceding $P$ wave is slightly but not markedly different from that seen in the previous figure. It will be noted in Figure 2 as well as in the other figures that the ventricular complexes following long periods of ventricular standstill differ somewhat in form from the others. This difference is partly due to the prolongation of the $\mathrm{R}-\mathrm{T}$ time and is probably analngous to the changes in the form of the ventricular complex which occur as a result of changes in heart rate.

Records obtained shortly after Figure 2, show in addition to the abnormal ventricular complexes single normal ventricular complexes interrupting the period of ventricular standstill. These normal beats 
bear the same relationship to the previous auricular beat as do the first ventricular beats after the long pauses.

Figure 3 is an example of the curves obtained shortly after Figure 2. The abnormal ventricular complexes no longer occur, but single normal ventricular complexes occur during the pauses at the rate of 1 for every 4 auricular beats, each ventricular complex bearing the same relationship to the preceding $P$ wave as the first ventricular complex of a group. During the pauses 4:1 block is present, while during the periods of rapid ventricular rate there is complete dissociation.

Figure 4 is an example of the electrocardiograms obtained about 4:05 p. m., May 10 . The groups of rapidly recurring ventricular beats no longer occur, and partial heart-block of high but somewhat mixed grade is now continuously present. Records obtained shortly after that shown in Figure 4 indicated a lower grade of partial block.

Figure 5 is an example of the curves obtained between 10:30 and 11 a. m., May 11 . This figure shows a return of complete dissociation. The ventricular rate, however, is now only 66 per minute, instead of 90 per minute as on the previous day. Moreover, there are no periods of ventricular standstill. At this time the patient was given one-fiftieth grain of atropin sulphate hypodermically.

Figure 6 was obtained seven minutes after the atropin was injected. We see in this figure a $2: 1$ block with a ventricular rate of 56 as compared with 66 in the previous figure. This $2: 1$ block continued without change for the next hour. In view of the fact that it came on so soon after the administration of the atropin and that there was no change in the block during the next hour, it seems unlikely that the atropin was responsible for the disappearance of the complete block seen in the previous figure in favor of partial block. On the other hand we believe that this was due to the slowing of the idioventricular rate.

On the following day, May 12, the partial block was of still lower grade, a mixture of $3: 2$, and $4: 3$ rhythm.

Figure 7 was taken at 10:45 a. m., May 14, four days after admission. Each auricular beat now produces a ventricular response, and the only sign of defective conduction is the prolongation of the P-R interval which measures from 0.310 to 0.315 second.

\section{DISCUSSION}

In discussing the cause of the periods of ventricular standstill and consequently of the syncopal attacks, we may first examine the tracings in the light of previous experience. Such periods of ventricular standstill are known to occur when complete heart-block is suddenly produced in the experimental animal, and also when a high grade of 

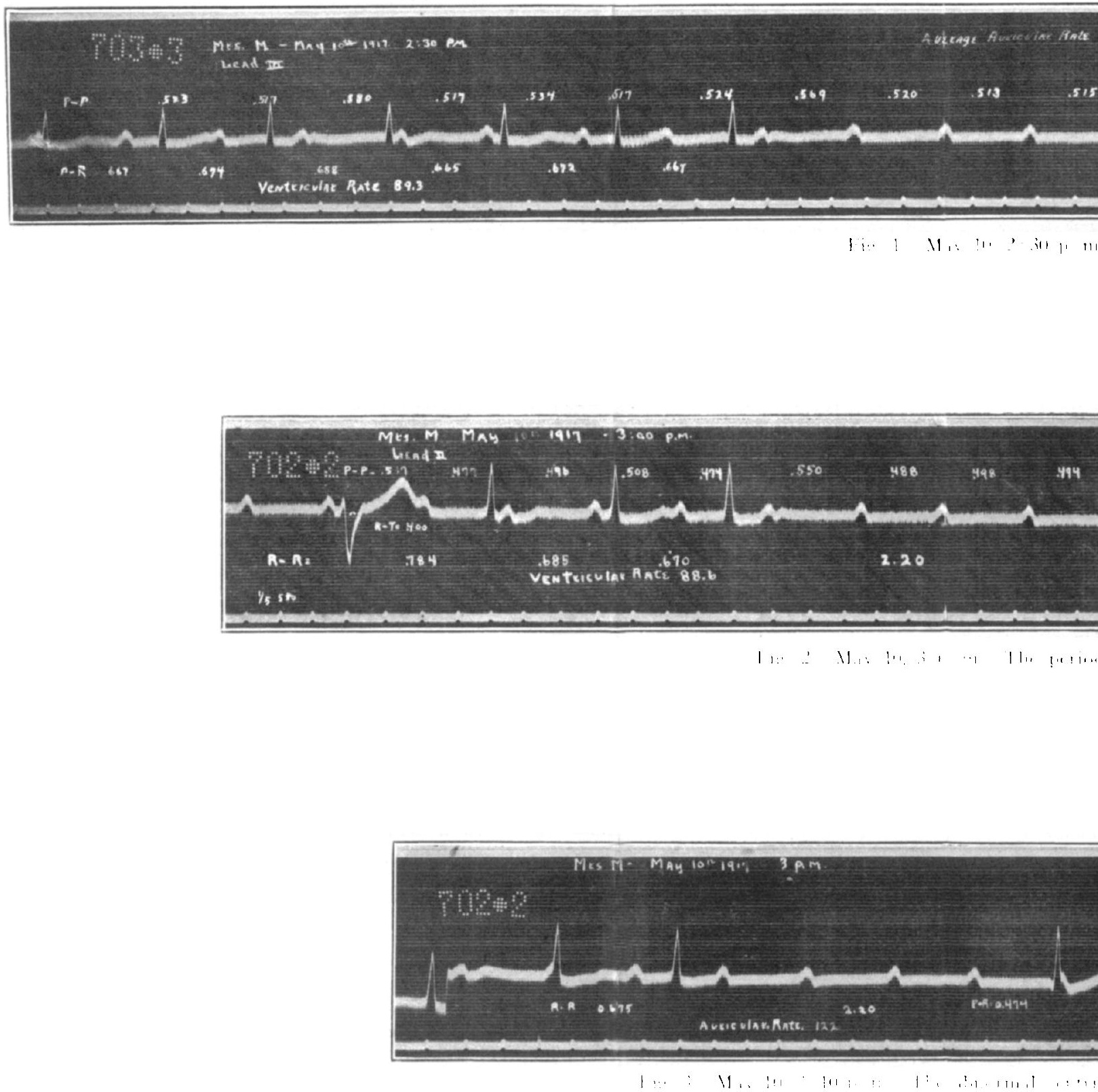

Downloaded From: http://archinte.jamanetwork.com/ by a Penn State Milton S Hershey Med Ctr User on 05/26/2015 


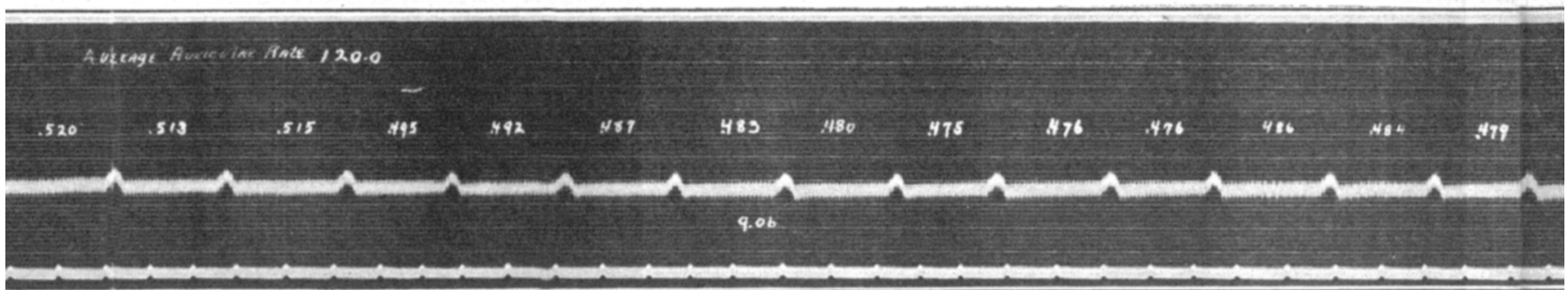

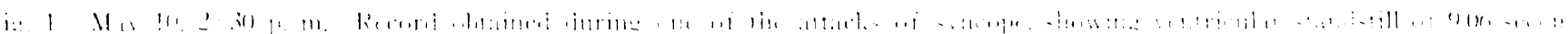

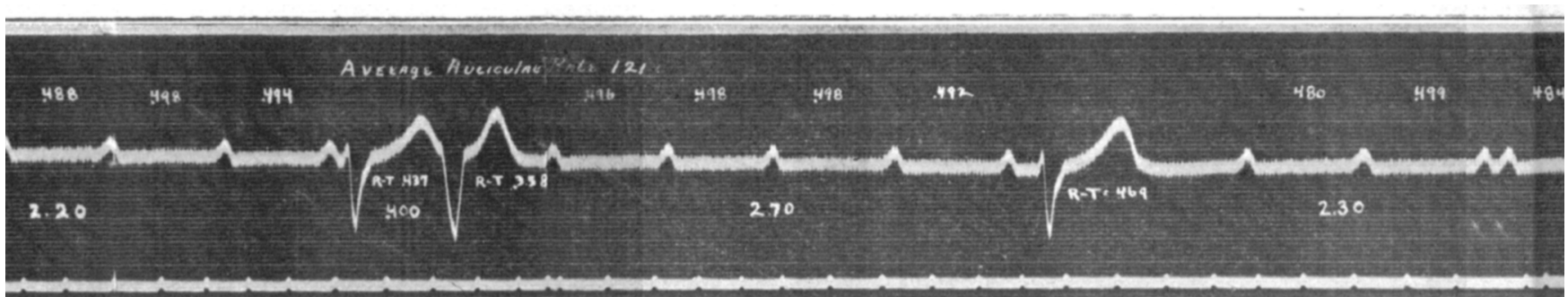

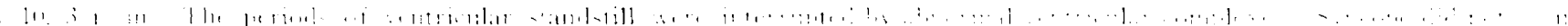

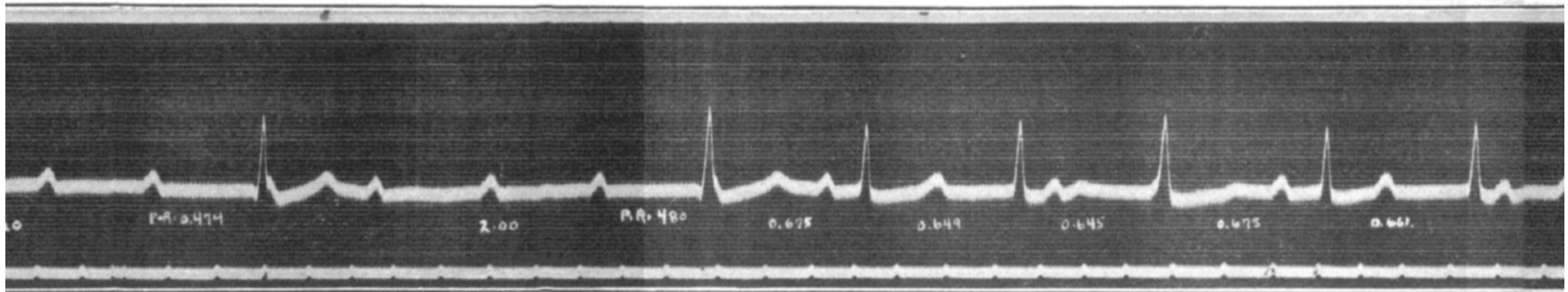

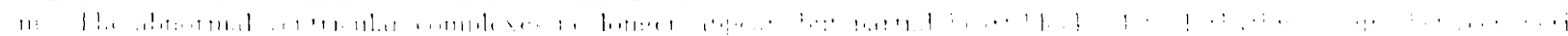

Downloaded From: http://archinte.jamanetwork.com/ by a Penn State Milton S Hershey Med Ctr User on 05/26/2015 


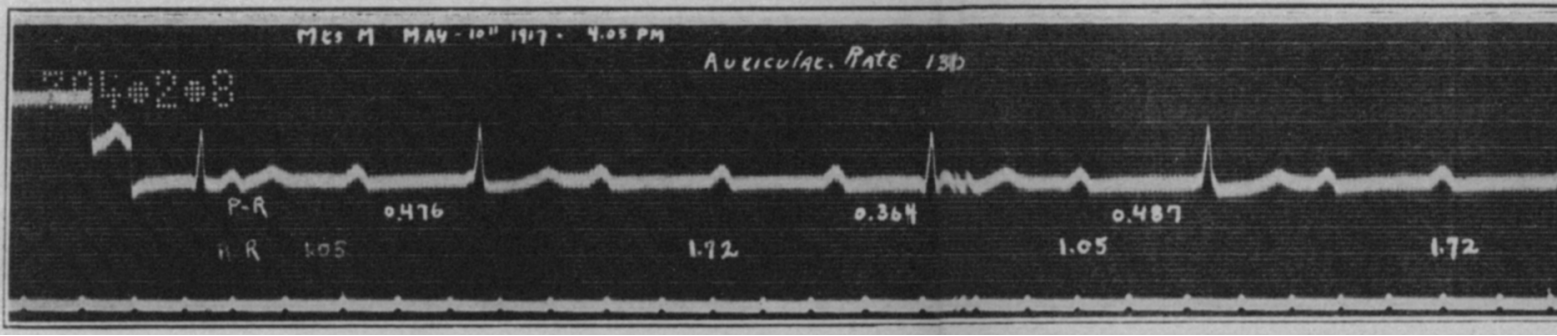

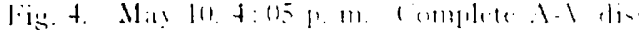

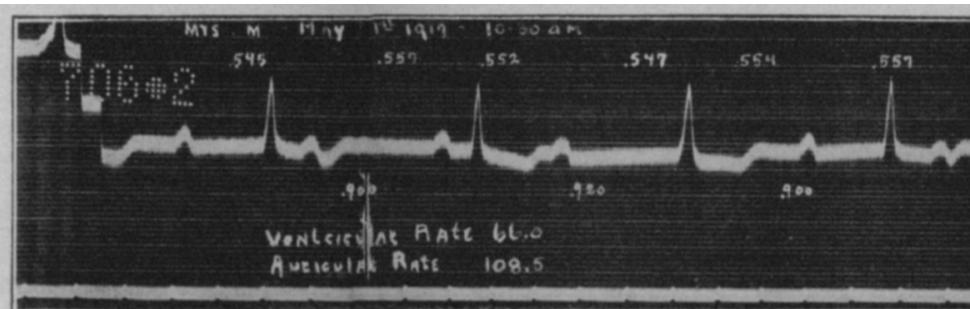

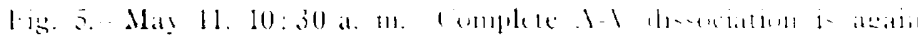

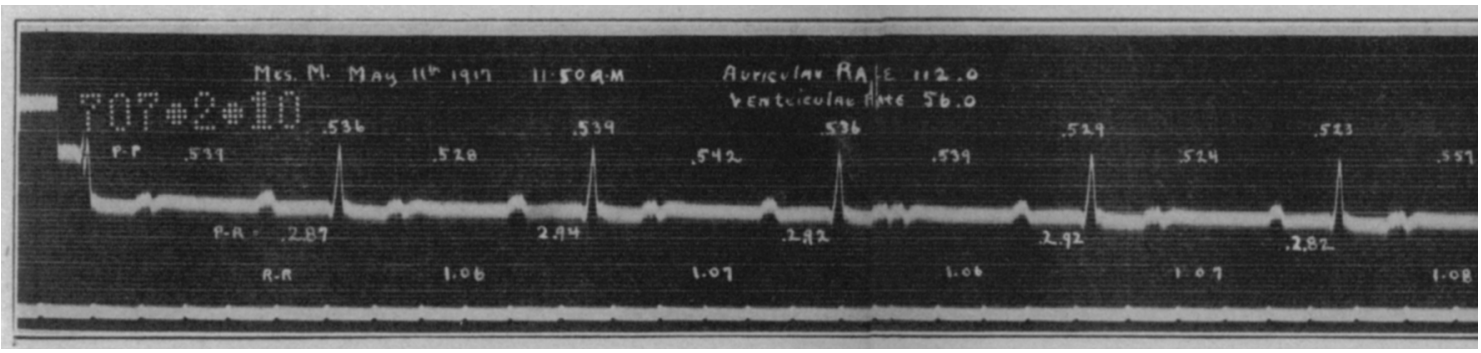

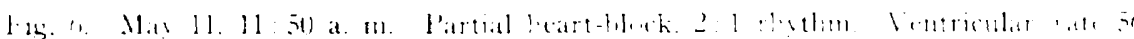




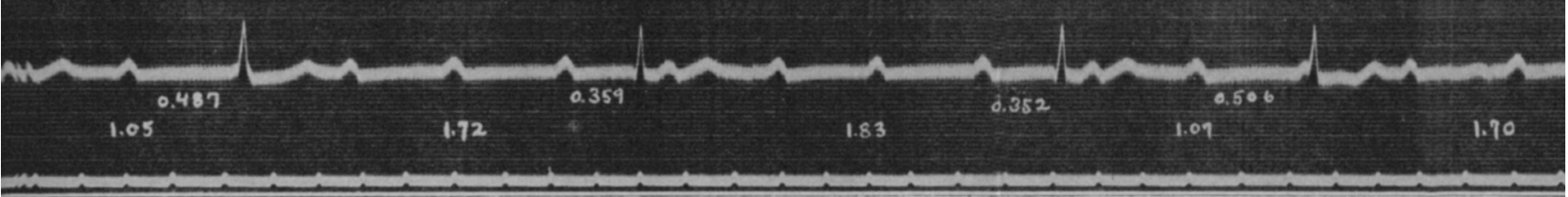

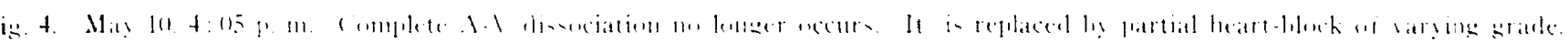

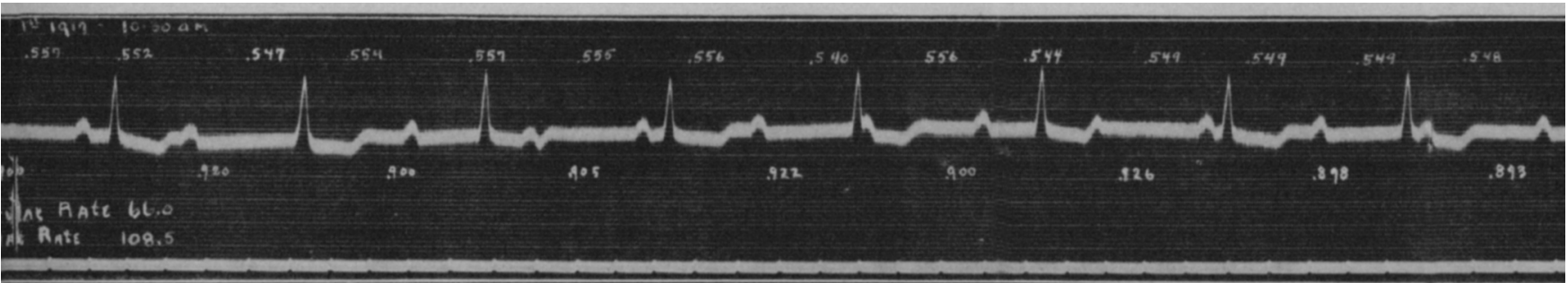

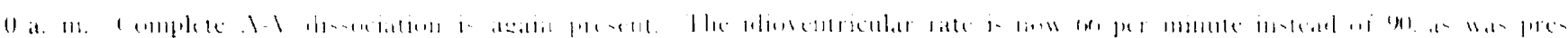
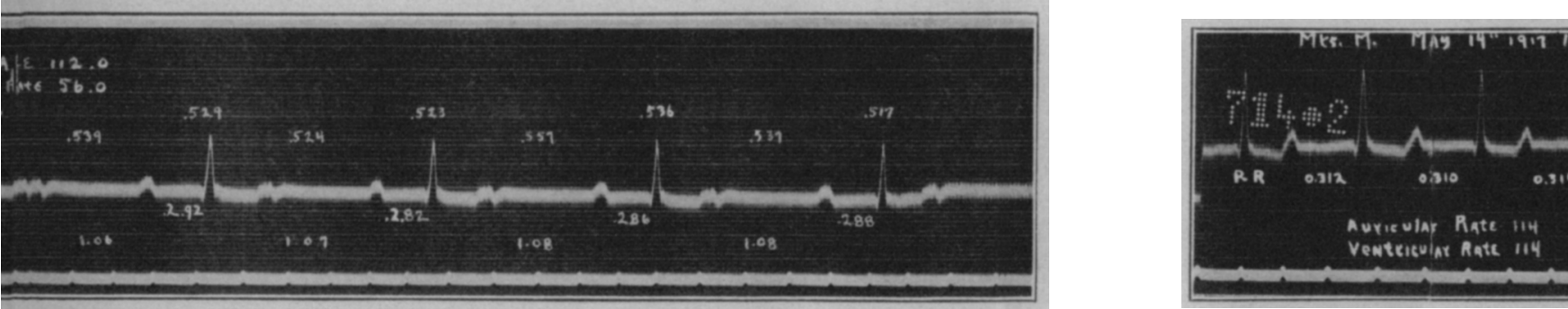

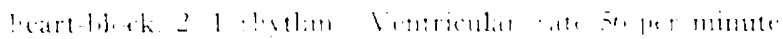

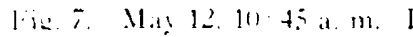


partial block suddenly becomes complete in man. In these instances ventricular stoppage is due, according to Erlanger and Hirschfelder, ${ }^{3}$ to the slow development of the idioventricular rhythm. In our case, however, none of the above conditions were present; we are dealing with very numerous periods of ventricular standstill occurring while complete block was continuously present. Moreover, the ventricles did not develop their rhythm gradually at the end of a period of standstill but started in at full speed. In looking for unusual features which might explain the ventricular stoppage the attention is attracted by the unusually rapid idioventricular rate, which was about three times as fast as the usual idioventricular rate seen in complete heart-block. This seems to offer an analogy. The ventricular tachycardia in this case bears certain resemblances to a well recognized type of tachycardia, namely, paroxysmal tachycardia, which is characterized by the suddenness of its termination. In some cases of tachycardia there is perhaps a causal relationship between the successive beats of the paroxysm; each beat seems to be necessary for the production of the following beat. If for any reason any beat of the series fails to occur, the tachycardia comes to an end. Somewhat the same mechanism may be discerned during the periods of idioventricular tachycardia which occurred in our patient. Each period stopped suddenly without ascertainable cause.

The mechanism by which the ventricular stoppage was terminated became evident when a number of records were examined. It was found that the first ventricular beat following the long pauses bore a fairly constant relationship to a preceding auricular beat, indicating that these beats were sequential and that the ventricular stoppage was terminated because an impulse from the auricles reached the ventricles. The auricular impulse did not stimulate the ventricles, however, until the ventricular stoppage had continued sufficiently long to produce the conditions in the heart which were necessary for the effective passage of the impulse. In the later records, when conduction was somewhat improved, the periods of standstill were shorter.

It seems likely, therefore, that the ventricular stoppage was at least partly dependent on the abnormal character of the ventricular rhythm during the periods of ventricular tachycardia. The length of the periods of ventricular standstill was apparently determined by the time required for sufficient improvement in the auriculoventricular conductivity to allow an auricular stimulus to reach the ventricles; for, as the degree of block diminished the periods of ventricular standstill became shorter. The periods of standstill were apparently never long

3. Erlanger and Hirschfelder: Am. Jour. Physiol., 1905-1906, 15, 153. 
enough to allow the usual slow idioventricular rhythm to develop. Three factors, then, took part in the production of the syncopal attacks: the abnormal character of the ventricular rhythm, the high grade of heart-block, and the slow development of the idioventricular rhythm.

The cessation of the syncopal attacks was apparently due to the occurrence of ventricular contractions arising in the left ventricle during the long ventricular pauses. These beats, although they did not always occur after the elapse of exactly the same period of time following the occurrence of ventricular stoppage, were probably due to an impulse generated in one of the lower ventricular centers. Had these beats failed to occur, however, the attacks would soon have ceased because of the rapid improvement in $\mathrm{A}-\mathrm{V}$ conduction and the gradual disappearance of the tendency to ventricular tachycardia which are shown by the later records of May 10.

The occurrence of a period of complete dissociation, May 11, appears to have been due to the recurrence of a ventricular rhythm of high rate. This rhythm may or may not have been of the same character as that previously observed. The ventricular rate was considerably slower and there was apparently no tendency to ventricular stoppage at this time. This ventricular tachycardia disappeared rather suddenly after, but probably not as a result of, the administration of atropin, giving place to a $2: 1$ rhythm. During the $2: 1$ rhythm the ventricular rate was considerably slower and it is possible that it was the rapid ventricular rate and not the condition of the junctional tissues alone which was responsible for the previous complete dissociation.

Two explanations may be given for the occurrence of such a phenomenon. The rapidly recurring ventricular beats may in some way depress the conductivity of the His bundle. This idea is supported by the occurrence of prolonged P-R intervals after interpolated ventricular extrasystoles. Erlanger, ${ }^{4}$ however, has suggested a different explanation. His view may be expressed as follows: The irritability of the ventricles during the refractory period is zero; following the refractory phase the irritability of the ventricles increases according to a definite curve. The height to which the curve of irritability rises depends on the form of the curve and on the duration of ventricular diastole. The value of the threshold stimulus depends at any time, therefore, on the time which has elapsed since the previous ventricular beat and the rate of recovery of the ventricles. When, because of the high rhythmicity of some ventricular center, the ventricles are driven at a rapid rate, the general ventricular irritability never reaches a high level. It is conceivable that in such instances the stimuli from the auricles may fail to be effective on reaching the ventricles because they lie below the

4. Erlanger: Personal communication. 
threshold value. When, as in the present case, the ventricular rate is relatively slow when compared with other types of tachycardia, this explanation is inadequate unless we further assume that the strength of the auricular stimuli reaching the ventricles is diminished by the defective $\mathrm{A}-\mathrm{V}$ conduction, or that the increase in ventricular irritability is much less rapid than is usually the case. So far as we know, there is no exact experimental evidence bearing on this problem.

It remains to inquire into the etiology of the heart-block in our patient. The rapidity with which the block cleared up suggests that it was not due to some destructive lesion of the A-V bundle, but to some toxic or acute inflammatory process. The patient had received small doses of digitalis ( 5 drops of a proprietary preparation) for a period of about one week before entering the hospital; but it seemed unlikely that this small amount of the drug could have been responsible for the block unless the patient was especially susceptible to the digitalis bodies. To decide this point the tincture of digitalis was administered to the patient for a considerable period, beginning about two weeks after she entered the hospital. No marked changes in conduction were observed. The patient's history and the slight fever on the day of admission to the hospital indicate that she had recently had an acute infection, and it is possible that this was in some way responsible for the lesion which caused the block. The presence of an old endocarditis and possibly, a chronic myocarditis indicated by the history and the presence of mitral regurgitation may have been a contributory factor.

\section{SUM MARY}

A case of transient complete heart-block is reported. When first observed the patient was having a great many syncopal attacks and the cardiac mechanism during some of these was recorded electrocardiographically. The attacks were caused by ventricular standstill lasting from seven to eleven seconds. The patient made a rapid clinical recovery, and at the same time there was a rapid decrease in the grade of block. The cause of the syncopal attacks and the etiology of the heart-block are discussed. 